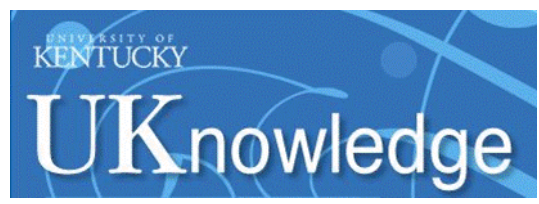

University of Kentucky

UKnowledge

\title{
Metal-Poor, Strongly Star-Forming Galaxies in the DEEP2 Survey: The Relationship Between Stellar Mass, Temperature-Based Metallicity, and Star Formation Rate
}

\author{
Chun Ly \\ NASA Goddard Space Flight Center \\ Jane R. Rigby \\ NASA Goddard Space Flight Center \\ Michael Cooper \\ University of California - Irvine \\ Renbin Yan \\ University of Kentucky, yanrenbin@uky.edu
}

Follow this and additional works at: https://uknowledge.uky.edu/physastron_facpub

Part of the Astrophysics and Astronomy Commons, and the Physics Commons

Right click to open a feedback form in a new tab to let us know how this document benefits you.

\section{Repository Citation}

Ly, Chun; Rigby, Jane R.; Cooper, Michael; and Yan, Renbin, "Metal-Poor, Strongly Star-Forming Galaxies in the DEEP2 Survey: The Relationship Between Stellar Mass, Temperature-Based Metallicity, and Star Formation Rate" (2015). Physics and Astronomy Faculty Publications. 331.

https://uknowledge.uky.edu/physastron_facpub/331

This Article is brought to you for free and open access by the Physics and Astronomy at UKnowledge. It has been accepted for inclusion in Physics and Astronomy Faculty Publications by an authorized administrator of UKnowledge. For more information, please contact UKnowledge@lsv.uky.edu. 


\section{Metal-Poor, Strongly Star-Forming Galaxies in the DEEP2 Survey: The Relationship Between Stellar Mass, Temperature-Based Metallicity, and Star Formation Rate}

Digital Object Identifier (DOI)

https://doi.org/10.1088/0004-637X/805/1/45

Notes/Citation Information

Published in The Astrophysical Journal, v. 805, no. 1, article 45, p. 1-10.

(c) 2015. The American Astronomical Society. All rights reserved.

The copyright holder has granted the permission for posting the article here.

This article is available at UKnowledge: https://uknowledge.uky.edu/physastron_facpub/331 


\title{
METAL-POOR, STRONGLY STAR-FORMING GALAXIES IN THE DEEP2 SURVEY: THE RELATIONSHIP BETWEEN STELLAR MASS, TEMPERATURE-BASED METALLICITY, AND STAR FORMATION RATE
}

\author{
Chun Ly ${ }^{1,4}$, Jane R. Rigby ${ }^{1}$, Michael CoOper ${ }^{2}$, and Renbin Yan ${ }^{3}$ \\ ${ }^{1}$ Observational Cosmology Laboratory, NASA Goddard Space Flight Center, 8800 Greenbelt Road, Greenbelt, MD 20771, USA; chun.ly@nasa.gov \\ ${ }^{2}$ Center for Galaxy Evolution, Department of Physics and Astronomy, UCI, Irvine, CA, USA \\ ${ }^{3}$ Department of Physics and Astronomy, University of Kentucky, Lexington, KY, USA \\ Received 2014 December 4; accepted 2015 March 11; published 2015 May 19
}

\begin{abstract}
We report on the discovery of $28 z \approx 0.8$ metal-poor galaxies in DEEP2. These galaxies were selected for their detection of the weak [O III] $\lambda 4363$ emission line, which provides a "direct" measure of the gas-phase metallicity. A primary goal for identifying these rare galaxies is to examine whether the fundamental metallicity relation (FMR) between stellar mass, gas metallicity, and star formation rate (SFR) holds for low stellar mass and high SFR galaxies. The FMR suggests that higher SFR galaxies have lower metallicity (at fixed stellar mass). To test this trend, we combine spectroscopic measurements of metallicity and dust-corrected SFR with stellar mass estimates from modeling the optical photometry. We find that these galaxies are $1.05 \pm 0.61$ dex above the $z \sim 1$ stellar mass-SFR relation and $0.23 \pm 0.23$ dex below the local mass-metallicity relation. Relative to the FMR, the latter offset is reduced to 0.01 dex, but significant dispersion remains $(0.29$ dex with 0.16 dex due to measurement uncertainties). This dispersion suggests that gas accretion, star formation, and chemical enrichment have not reached equilibrium in these galaxies. This is evident by their short stellar mass doubling timescale of $\approx 100_{-75}^{+310}$ Myr, which suggests stochastic star formation. Combining our sample with other $z \sim 1$ metal-poor galaxies, we find a weak positive SFR-metallicity dependence (at fixed stellar mass) that is significant at $94.4 \%$ confidence. We interpret this positive correlation as recent star formation that has enriched the gas but has not had time to drive the metal-enriched gas out with feedback mechanisms.
\end{abstract}

Key words: galaxies: abundances - galaxies: distances and redshifts - galaxies: evolution - galaxies: ISM galaxies: photometry - galaxies: starburst

\section{INTRODUCTION}

The chemical enrichment of galaxies, driven by star formation and modulated by gas flows from supernova and cosmic accretion, is key for understanding galaxy formation and evolution. The primary approach for measuring metal abundances is spectroscopy of nebular emission lines. These emission lines can be observed in the optical and near-infrared at $z \lesssim 3$ from the ground (e.g., Kobulnicky \& Kewley 2004; Erb et al. 2006; Maiolino et al. 2008; Hainline et al. 2009; Hayashi et al. 2009; Mannucci et al. 2009; Moustakas et al. 2011; Rigby et al. 2011; Zahid et al. 2011; Nakajima et al. 2012; Henry et al. 2013a; Momcheva et al. 2013; Pirzkal et al. 2013; Ly et al. 2014; Troncoso et al. 2014; de los Reyes et al. 2015) and space (e.g., Atek et al. 2011; van der Wel et al. 2011; Xia et al. 2012; Henry et al. 2013b; Whitaker et al. 2014b), and the James Webb Space Telescope will extend this to $z \sim 6$.

The most reliable metallicity measurements are made by measuring the flux ratio of the $[\mathrm{O}$ III $] \lambda 4363$ line against $[\mathrm{O}$ III] 15007. The technique is called the $T_{e}$ method because it determines the electron temperature $\left(T_{e}\right)$ of the gas, and hence the gas-phase metallicity (Aller 1984). However, the detection of [O $\mathrm{III}] \lambda 4363$ is difficult, as it is weak, almost undetectable in metal-rich galaxies. Only 0.3\% of the Sloan Digital Sky Survey (SDSS) has detected [O III] $\lambda 4363$ at signal-to-noise ratio $(\mathrm{S} / \mathrm{N}) \geqslant 2$ (Izotov et al. 2006).

Efforts have been made to increase the number of galaxies with direct metallicities in the local universe (e.g., Brown et al. 2008; Berg et al. 2012; Izotov et al. 2012) and at $z \gtrsim 0.2$

\footnotetext{
${ }^{4}$ NASA Postdoctoral Fellow.
}

(Hoyos et al. 2005; Kakazu et al. 2007; Hu et al. 2009; Amorín et al. 2014a, 2014b; Ly et al. 2014); however, the total sample size is $\sim 145$ (mostly in the local universe).

Using the DEEP2 Galaxy Redshift Survey (Davis et al. 2003; Newman et al. 2013), we are conducting studies that utilize $[\mathrm{O}$ III $] \lambda 4363$ detections in individual galaxies and from stacked spectra. In this paper, we focus on first results from individual [O $\mathrm{OII}] \lambda 4363$ detections in 28 galaxies. Our sample of 28 galaxies substantially increases the number of $z \geqslant 0.25$ galaxies with $\mathrm{S} / \mathrm{N} \geqslant 3[\mathrm{O}$ III] $\lambda 4363$ detections by $44 \%$ (from 64 to 92).

While the selection function is biased toward metal-poor galaxies, this galaxy population is of significant interest for understanding galaxy evolution. Their low metallicity suggests that they are either (1) in their earliest stages of formation, (2) accreting metal-poor gas, or (3) undergoing significant metalenriched gas outflows. Metal-poor galaxies have been poorly studied due to the difficulty of identifying them. Thus, the majority of previous high- $z$ metallicity studies have only used strong-line metallicity calibrations. These calibrations are problematic for high- $z$ galaxies due to suspected differences in the physical conditions of the interstellar gas (e.g., Liu et al. 2008; Kewley et al. 2013a, 2013b), but see also Juneau et al. (2014) for a different interpretation. These differences, if present, may be incorrectly interpreted as evolution in the metal content. Thus, obtaining $T_{e}$-based metallicities at high redshifts is the next logical step, and these [O III] $\lambda 4363$ detections can potentially be used to recalibrate the strong-line diagnostics.

While the $T_{e}$ method is affected by properties of the ionized gas (e.g., optical depth, density, ionization parameter, nonequilibrium electron energy; Nicholls et al. 2014), such effects 
also apply to strong-line diagnostics as Nicholls et al. (2014) demonstrated. Thus, while the $T_{e}$ method is less "direct" than was initially determined (Seaton 1954), measuring the electron temperature currently remains the preferred way to determine gas metallicities.

In this paper, we focus on the relationship between stellar mass, gas-phase metallicity, and star formation rate (SFR) for our sample of 28 metal-poor galaxies. This relationship has received significant interest as Ellison et al. (2008) found that at a given stellar mass, lower-metallicity galaxies in the local universe tend to have higher SFRs. Thus, while the stellar mass-metallicity relation is tight $(\sim 0.1 \mathrm{dex}$; Tremonti et al. 2004), it may be a projection of a non-evolving threedimensional relationship between stellar mass $\left(M_{\star}\right)$, gas-phase metallicity (Z), and SFR (e.g., Lara-López et al. 2010; Mannucci et al. 2010; Hunt et al. 2012).

However, the existence of an $M_{\star}-Z-S F R$ relation (dubbed the "fundamental metallicity relation" or "FMR") remains controversial, as recent studies have yielded results that agree or disagree with predictions (see Salim et al. 2014; de los Reyes et al. 2015, for a review). Moreover, the $M_{\star}-Z-S F R$ relation has yet to be tested with large samples of metal-poor $\left(Z \lesssim 0.25 Z_{\odot}\right)$ galaxies, especially at higher redshift. The largest high- $z$ metal-poor sample is that of Ly et al. (2014) from the Subaru Deep Field (SDF; Kashikawa et al. 2004; Ly et al. 2007), which detected [O III] $\lambda 4363$ in 20 galaxies at $z \sim 0.4-1$. In this study, they found evidence that galaxies with the highest specific SFR $\left(\mathrm{SFR} / M_{\star}\right.$, hereafter sSFR) were not necessarily more metal-poor. This result, based on 20 galaxies, requires further confirmation with our DEEP2 sample.

In this study, we compare our work with Andrews \& Martini (2013) who stacked $0.027<z<0.25$ SDSS spectra in bins of SFR and $M_{\star}$ to obtain average [O III] $\lambda 4363$ measurements. We note that the primary selection functions of Andrews \& Martini (2013) (magnitude-limited) and our study (emission-line strengths) are different. In a forthcoming paper, we will follow the same approach as Andrews \& Martini (2013) of stacking the spectra of a few thousand $z \sim 0.8$ DEEP2 galaxies, allowing for a more self-consistent comparison. For now, we investigate the $M_{\star}-Z-\mathrm{SFR}$ relation with individual $T_{e}$ metallicity measurements. Throughout this paper, we adopt a cosmology with $\Omega_{\Lambda}=0.7, \Omega_{M}=0.3$, and $h=0.7$, a Chabrier (2003) initial mass function (IMF), and a solar metallicity of $12+\log (\mathrm{O} / \mathrm{H})=8.69$.

\section{THE SAMPLE}

The DEEP2 Survey has surveyed $\sim 3 \mathrm{deg}^{2}$ over four fields using the DEIMOS multi-object spectrograph (Faber et al. 2003) on the Keck II telescope. The survey has provided optical ( $\approx 6500-9000 \AA$ ) spectra for $\sim 53,000$ galaxies brighter than $R_{\mathrm{AB}}=24.1$ and precise redshifts for $\sim 70 \%$ of targeted galaxies. An overview of the survey can be found in Newman et al. (2013).

Using the fourth data release (DR4), ${ }^{5}$ we select 37,396 sources with reliable redshifts (quality flag $\geqslant 3$ ). We consider those with spectral coverage that spans $3720-5010 \AA$ (rest frame). This enable us to determine metallicity from oxygen and hydrogen emission lines ([O $\mathrm{II}] \lambda \lambda 3726,3729,[\mathrm{O}$ III $] \lambda \lambda \lambda$ $4363,4959,5007$, and $\mathrm{H} \beta$ ) and further limits the sample to 4140 galaxies at $z=0.697-0.859$ (average: 0.779 ).

\footnotetext{
http://deep.ps.uci.edu/dr4/home.htm
}

We follow the approach of Ly et al. (2014) that fits emission lines with Gaussian profiles using the IDL routine MPFIT (Markwardt 2009). Spectroscopic redshifts are used as priors for the location of emission lines. With measurements of emission-line fluxes and the noise in the spectra (measured from a $200 \AA$ region around each line), we select those with [O III $] \lambda 4363$ and $[\mathrm{O}$ III $] \lambda 5007$ detected at $\mathrm{S} / \mathrm{N} \geqslant 3$ and $\mathrm{S} / \mathrm{N} \geqslant$ 100 , respectively. This yields an initial sample of 54 galaxies. We inspect each spectrum and remove 26 galaxies from our sample, primarily because of contamination from $\mathrm{OH}$ skylines. This leaves 28 galaxies. One source (\#21) was observed twice. The other spectrum also detected $[\mathrm{O}$ III $] \lambda 4363$ at lower $\mathrm{S} / \mathrm{N}$, so the better spectrum is used in our analysis. Compared to the previous DEEP2 sample (Hoyos et al. 2005), we confirmed two; thus, 26 galaxies in our sample are newly identified.

Detections of [O III] $\lambda 4363$ are shown in Figure 1, and galaxy properties are provided in Table 1 . We illustrate in Figure 2 the emission-line luminosities, rest-frame equivalent widths (EWs), and $O_{32} \equiv[\mathrm{O} \mathrm{III}] /[\mathrm{O}$ II $]$ and $R_{23} \equiv\left(\left[\begin{array}{ll}\mathrm{OI}\end{array}\right]+\right.$ $[\mathrm{O}$ III $]) / \mathrm{H} \beta$ flux ratios (Pagel et al. 1979; McGaugh 1991) and compare our sample to local galaxies and other [O III] $\lambda 4363$ detected galaxies (Ly et al. 2014).

\subsection{Flux Calibration}

The publicly released data of DEEP2 are not flux-calibrated, which is problematic for measuring the 4363 to 5007 ratio and hence $T_{e}$. To address this limitation, we use proprietary IDL codes developed by Jeffrey Newman, Adam Walker, and Renbin Yan of the DEEP2 team. These codes take into account the overall throughput, quantum efficiency of the eight CCD detectors, apply coarse telluric corrections for atmospheric absorption bands, and use the $R$ and $I$ DEEP2 photometry to transform the spectrum to energy units. The DEEP2 team has demonstrated that the calibration is reliable at the $10 \%$ level when compared to SDSS stars observed by DEEP2.

\section{DERIVED PROPERTIES}

\subsection{Dust Attenuation Correction from Balmer Decrements}

To correct the emission-line fluxes for dust attenuation, we use Balmer decrement measurements. At $z \sim 0.8$, the existing DEEP2 optical spectra measure $\mathrm{H} \beta, \mathrm{H} \gamma$, and $\mathrm{H} \delta$. While these lines are intrinsically weak compared to $\mathrm{H} \alpha,{ }^{6}$ our galaxies possess high emission-line EWs, which result in 22, 26, and 28 galaxies having $\mathrm{H} \delta, \mathrm{H} \gamma$, and $\mathrm{H} \beta$ detected at $\mathrm{S} / \mathrm{N} \geqslant 10$, respectively. The significant detections enable dust attenuation measurements of $\sigma(\mathrm{A}(\mathrm{H} \alpha)) \approx 0.1 \mathrm{mag}$ (average from $\mathrm{H} \gamma / \mathrm{H} \beta$ ).

A problem encountered with Balmer emission lines is the underlying stellar absorption. Our examination of each spectrum reveals weak stellar absorption, making it difficult to obtain reliable fits to the broad wings of absorption lines. To address this limitation, we stack our spectra. Here, the continuum (around each Balmer line) is normalized to one, and an average is computed with the exclusion of spectral regions affected by $\mathrm{OH}$ sky-line emission. Stellar absorption is detected in $\mathrm{H} \delta$ and is consistent with an $\mathrm{EW}_{\text {rest }}$ correction of $1 \AA$. For our entire sample, we adopt an $\mathrm{EW}_{\text {rest }}$ correction of $1 \AA$ for $\mathrm{H} \beta \mathrm{H} \gamma$, and $\mathrm{H} \delta$. With these corrections for stellar absorption, we illustrate the Balmer decrements in Figure 3.

\footnotetext{
${ }^{6} \mathrm{H} \alpha$ is redshifted beyond the optical spectral coverage.
} 


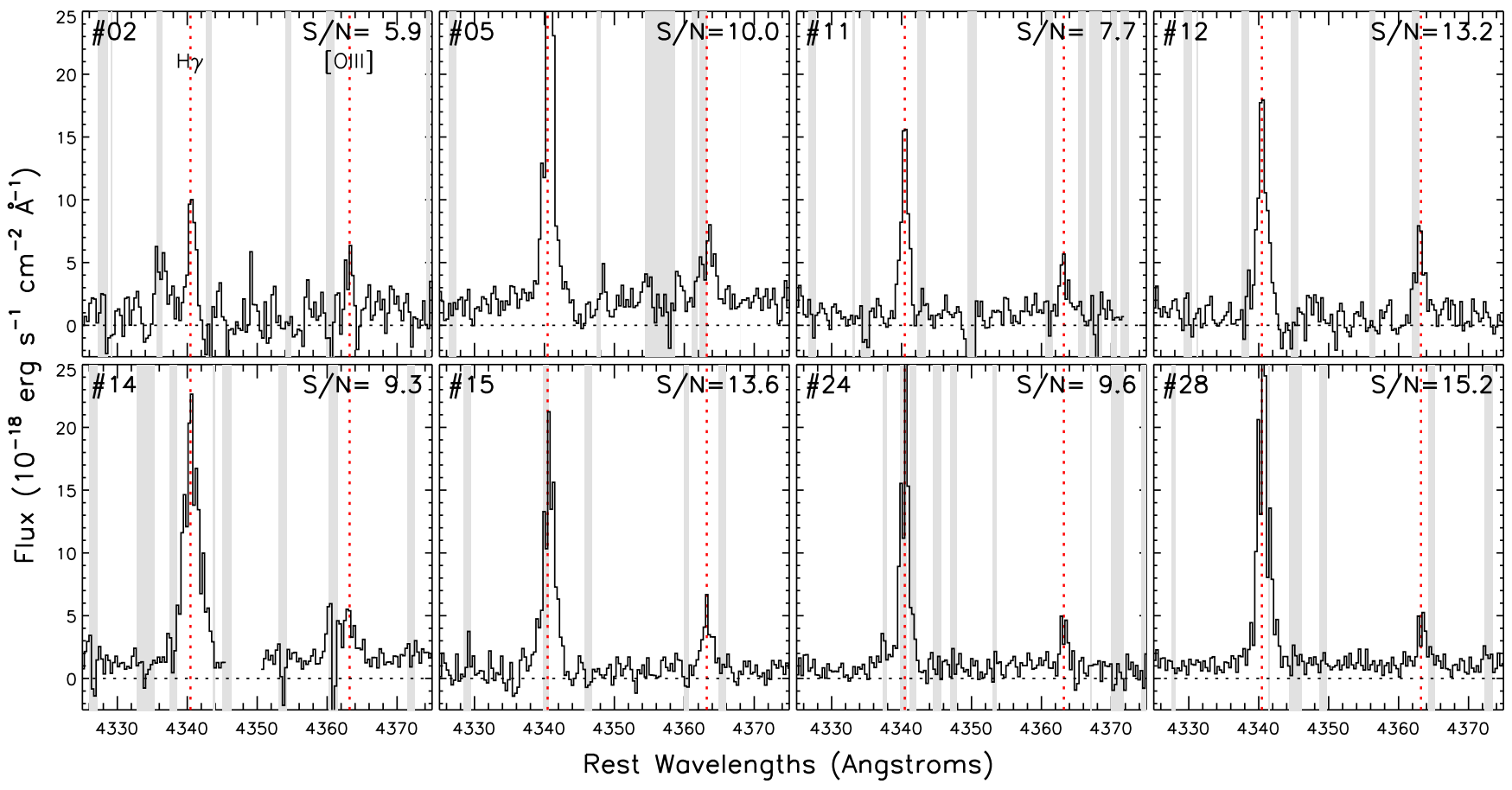

Figure 1. Detections of [O III] $\lambda 4363$ in $z \sim 0.8$ DEEP2 galaxies. The Keck/DEIMOS spectra for 8 of 28 galaxies are shown by the solid black lines, with vertical red dashed lines indicating the locations of $\mathrm{H} \gamma \lambda 4340$ and $[\mathrm{O} \mathrm{III}] \lambda 4363$. OH skylines are indicated by the gray shaded regions. The $\mathrm{S} / \mathrm{N}$ of $[\mathrm{O}$ III] $\lambda 4363 \mathrm{detections}$ is reported in the top right.

Assuming that the hydrogen emission originates from an optically thick $\mathrm{H}$ II region obeying Case $\mathrm{B}$ recombination, the intrinsic Balmer flux ratios are $(\mathrm{H} \gamma / \mathrm{H} \beta)_{0}=0.468$ and $(\mathrm{H} \delta /$ $\mathrm{H} \beta)_{0}=0.259$. Dust absorption alters these observed ratios as follows:

$$
\frac{(\mathrm{H} n / \mathrm{H} \beta)_{\text {obs }}}{(\mathrm{H} n / \mathrm{H} \beta)_{0}}=10^{-0.4 E(B-V)[k(\mathrm{H} n)-k(\mathrm{H} \beta)]},
$$

where $E(B-V)$ is the nebular color excess and $k(\lambda) \equiv A(\lambda) / E$ $(B-V)$ is the dust reddening curve. We illustrate in Figure 3 the observed Balmer decrements under the Calzetti et al. (2000) dust reddening formalism. We find that our Balmer decrements are consistent with Calzetti et al. (2000). For the remainder of the paper, all dust-corrected measurements adopt Calzetti et al. (2000) reddening.

Our color excesses are determined mostly (20/28) from $\mathrm{H} \gamma /$ $\mathrm{H} \beta$. For five galaxies, we use $\mathrm{H} \delta / \mathrm{H} \beta$ since $\mathrm{H} \gamma$ suffers from contamination from $\mathrm{OH}$ skylines. For the remaining three galaxies, the dust reddening could not be determined from either Balmer decrement (they were both affected by $\mathrm{OH}$ skyline emission). For these galaxies, we assume $E(B-V)=0.22 \pm 0.23 \mathrm{mag} \quad(A(\mathrm{H} \alpha) \approx 0.73 \pm 0.75 \mathrm{mag})$, which is the average of our sample and is consistent with other emission-line galaxy samples (e.g., Ly et al. 2012a; Domínguez et al. 2013; Momcheva et al. 2013). For Balmer decrements that imply negative reddening (six cases), we adopt $E(B-V)=0$ with measurement uncertainties based on Balmer decrement uncertainties.

\section{2. $T_{e}$-based Metallicity Determinations}

To determine the gas-phase metallicity for our galaxies, we follow previous direct metallicity studies and use the empirical relations of Izotov et al. (2006). Here, we briefly summarize the approach, and we refer readers to Ly et al. (2014) for more details. First, the $\mathrm{O}^{++}$electron temperature, $T_{e}([\mathrm{O}$ III $])$, can be estimated using the nebular-to-auroral $[\mathrm{O} \quad \mathrm{III}]$ ratio, $[\mathrm{O} \quad \mathrm{III}] \lambda \lambda$ $4959,5007 /[\mathrm{O}$ III $] \lambda 4363$. We correct the above flux ratio for dust attenuation (Section 3.1). We also apply a 5\% correction, since $T_{e}$ determinations from Izotov et al. (2006) are found to be overestimated (Nicholls et al. 2013).

Our $[\mathrm{O}$ III] measurements have a very large dynamic range. The strongest (weakest) $[\mathrm{O}$ III $\lambda 4363$ line is $6.5 \%(0.7 \%)$ of the $[\mathrm{O}$ III $] \lambda 5007$ flux. We find that the average (median) $\lambda 4363 / \lambda 5007$ flux ratio for our sample is $0.018(0.015)$. The derived $T_{e}$ for our galaxy sample spans $(1-3.1) \times 10^{4} \mathrm{~K}$.

To determine the ionic abundances of oxygen, we use two emission-line flux ratios, $[\mathrm{O}$ II $] \lambda \lambda 3726,3729 / \mathrm{H} \beta$ and $[\mathrm{O}$ III] $\lambda \lambda$ $4959,5007 / \mathrm{H} \beta$. For our metallicity estimation, we adopt a standard two-zone temperature model with $T_{e}\left(\left[\begin{array}{ll}\mathrm{O} & \mathrm{II}\end{array}\right]\right)=$ $0.7 T_{e}([\mathrm{O}$ III $])+3000$ (Andrews \& Martini 2013), to enable direct comparisons to local studies. In computing $\mathrm{O}^{+} / \mathrm{H}^{+}$, we also correct the $[\mathrm{O} \mathrm{II}] / \mathrm{H} \beta$ ratio for dust attenuation. We do not correct $[\mathrm{O} \mathrm{III}] / \mathrm{H} \beta$ since the effects are negligible.

Since the most abundant ions of oxygen in $\mathrm{H}$ II regions are $\mathrm{O}^{+}$and $\mathrm{O}^{++}$, the oxygen abundances are given by $\mathrm{O} / \mathrm{H}=\left(\mathrm{O}^{+}+\mathrm{O}^{++}\right) / \mathrm{H}^{+}$. In Table 1 , we provide estimates of $T_{e}\left(\left[\begin{array}{ll}\mathrm{O} & \mathrm{III}\end{array}\right]\right)$ and de-reddened metallicity for our sample. Our most metal-poor systems are \#04 and \#08 and can be classified as extremely metal-poor galaxies $\left(\leqslant 0.1 Z_{\odot}\right)$.

\subsection{Dust-corrected SFRs}

In addition to gas-phase metallicity, our data allow us to determine dust-corrected SFRs using the hydrogen recombination lines, which are sensitive to the shortest timescale of star formation, $\lesssim 10 \mathrm{Myr}$. 
Table 1

Summary of DEEP2 Sample

\begin{tabular}{|c|c|c|c|c|c|c|c|c|c|c|c|c|c|}
\hline ID & $\begin{array}{c}\text { R.A. } \\
\text { (hh:mm:ss) }\end{array}$ & $\begin{array}{c}\text { Decl. } \\
\text { (dd:mm:ss) }\end{array}$ & $z$ & $\begin{array}{c}\mathrm{EW}(\mathrm{H} \beta) \\
(\AA)\end{array}$ & $\log \left(M_{\star} / M_{\odot}\right)$ & $\log \left(\frac{\mathrm{SFRH} \beta}{M_{\odot} \mathrm{yr}^{-1}}\right)$ & $\begin{array}{c}E(B-V) \\
(\mathrm{mag})\end{array}$ & $\begin{array}{c}\mathrm{S} / \mathrm{N} \\
(\lambda 4363)\end{array}$ & {$[\mathrm{O} \quad \mathrm{II}] / \mathrm{H} \beta$} & {$[\mathrm{O} \mathrm{III}] / \mathrm{H} \beta$} & $\frac{[\mathrm{OIII}]}{[\mathrm{OIII}] \lambda 4363}$ & $\log \left(T_{e} / \mathrm{K}\right)$ & $12+\log (\mathrm{O} / \mathrm{H})$ \\
\hline 01 & $14: 18: 31.260$ & $52: 49: 42.545$ & 0.8194 & 38.46 & $8.81_{-0.00}^{+0.18}$ & $0.23 \pm 0.16$ & $0.00_{-0.08}^{+0.09}$ & 4.9 & $2.165_{-0.051}^{+0.101}$ & $5.507_{-0.067}^{+0.156}$ & $57.273_{-08.811}^{+11.748}$ & $4.18 \pm 0.04$ & $7.96_{-0.14}^{+0.11}$ \\
\hline 02 & $14: 21: 21.513$ & 53:01:07.672 & 0.7496 & 51.94 & $8.76_{-0.06}^{+0.04}$ & $0.54_{-0.33}^{+0.45}$ & $0.28_{-0.18}^{+0.24}$ & 5.9 & $1.821_{-0.235}^{+0.705}$ & $7.792_{-0.351}^{+0.200}$ & $67.843_{-06.619}^{+19.956}$ & $4.14 \pm 0.04$ & $8.13 \pm 0.10$ \\
\hline 03 & $14: 21: 25.487$ & 53:09:48.071 & 0.7099 & 104.49 & $8.94_{-0.15}^{+0.00}$ & $-0.09_{-0.25}^{+0.19}$ & $0.00_{-0.14}^{+0.10}$ & 3.7 & $2.413 \pm 0.103$ & $5.178_{-0.085}^{+0.198}$ & $54.985_{-10.473}^{+20.947}$ & $4.17_{-0.05}^{+0.06}$ & $7.98_{-0.19}^{+0.17}$ \\
\hline 04 & 14:22:03.718 & $53: 25: 47.766$ & 0.7878 & 7.38 & $\ldots$ & $-0.06_{-0.59}^{+0.63}$ & $0.00_{-0.32}^{+0.34}$ & 5.5 & $0.867 \pm 0.094$ & $7.273_{-0.578}^{+0.385}$ & $18.192_{-01.692}^{+0.523}$ & $4.49_{-0.07}^{+0.08}$ & $7.35_{-0.17}^{+0.11}$ \\
\hline 05 & $14: 21: 45.408$ & 53:23:52.699 & 0.7710 & 74.51 & $8.86_{-0.00}^{+0.07}$ & $1.50_{-0.05}^{+0.07}$ & $0.47_{-0.03}^{+0.04}$ & 10.0 & $1.960 \pm 0.084$ & $8.497_{-0.053}^{+0.080}$ & $90.482_{-08.133}^{+10.167}$ & $4.10 \pm 0.02$ & $8.27_{-0.06}^{+0.07}$ \\
\hline 06 & $16: 47: 26.188$ & $34: 45: 12.126$ & 0.7166 & 36.91 & $8.85_{-0.01}^{+0.12}$ & $0.71_{-0.10}^{+0.07}$ & $0.21_{-0.05}^{+0.04}$ & 5.0 & $2.539_{-0.095}^{+0.221}$ & $6.245 \pm 0.081$ & $131.237_{-15.908}^{+47.723}$ & $4.02_{-0.03}^{+0.02}$ & $8.51_{-0.10}^{+0.13}$ \\
\hline 07 & $16: 46: 35.420$ & 34:50:27.928 & 0.7624 & 34.71 & $9.07_{-0.00}^{+0.19}$ & $0.84_{-0.10}^{+0.12}$ & $0.22_{-0.05}^{+0.07}$ & 3.4 & $3.304_{-0.260}^{+0.208}$ & $5.003_{-0.081}^{+0.040}$ & $83.493_{-12.845}^{+25.690}$ & $4.09 \pm 0.05$ & $8.29_{-0.21}^{+0.15}$ \\
\hline 08 & $16: 47: 26.488$ & 34:54:09.770 & 0.7653 & 79.33 & $8.07_{-0.14}^{+0.69}$ & $0.15 \pm 0.42$ & $0.22 \pm 0.23$ & 4.0 & $1.412_{-0.097}^{+0.779}$ & $7.866_{-0.168}^{+0.589}$ & $24.249_{-04.217}^{+08.434}$ & $4.38_{-0.11}^{+0.08}$ & $7.63_{-0.21}^{+0.17}$ \\
\hline 09 & 16:49:51.368 & $34: 45: 18.210$ & 0.7909 & 53.06 & $9.00_{-0.32}^{+0.08}$ & $0.22_{-0.12}^{+0.17}$ & $0.02_{-0.07}^{+0.09}$ & 3.6 & $2.496_{-0.079}^{+0.197}$ & $5.485_{-0.099}^{+0.124}$ & $78.655_{-14.982}^{+29.964}$ & $4.10_{-0.04}^{+0.05}$ & $8.23_{-0.17}^{+0.16}$ \\
\hline 10 & $16: 51: 31.472$ & $34: 53: 15.964$ & 0.7945 & 64.52 & $8.70_{-0.31}^{+0.15}$ & $1.06 \pm 0.08$ & $0.31 \pm 0.04$ & 6.4 & $2.159_{-0.140}^{+0.093}$ & $6.739_{-0.040}^{+0.094}$ & $85.109_{-12.379}^{+15.474}$ & $4.11_{-0.03}^{+0.02}$ & $8.21_{-0.08}^{+0.09}$ \\
\hline 11 & 16:50:55.342 & $34: 53: 29.875$ & 0.7980 & 94.85 & $8.56_{-0.59}^{+0.21}$ & $0.11_{-0.11}^{+0.16}$ & $0.00_{-0.06}^{+0.08}$ & 7.7 & $2.086 \pm 0.065$ & $7.480_{-0.118}^{+0.148}$ & $58.301_{-11.181}^{+03.195}$ & $4.19 \pm 0.03$ & $7.97_{-0.11}^{+0.06}$ \\
\hline 12 & $16: 53: 03.486$ & 34:58:48.946 & 0.7488 & 69.05 & $8.33_{-0.08}^{+0.16}$ & $0.31_{-0.15}^{+0.10}$ & $0.06_{-0.08}^{+0.05}$ & 13.2 & $1.702_{-0.000}^{+0.229}$ & $6.384_{-0.056}^{+0.130}$ & $70.872_{-05.956}^{+04.765}$ & $4.15_{-0.01}^{+0.02}$ & $8.04_{-0.05}^{+0.03}$ \\
\hline 13 & $16: 51: 24.060$ & $35: 01: 38.740$ & 0.7936 & 32.65 & $9.09_{-0.35}^{+0.09}$ & $0.40_{-0.12}^{+0.17}$ & $0.23_{-0.06}^{+0.09}$ & 5.2 & $2.862_{-0.185}^{+0.369}$ & $6.241_{-0.103}^{+0.154}$ & $74.343_{-11.437}^{+15.250}$ & $4.13_{-0.04}^{+0.03}$ & $8.20_{-0.11}^{+0.12}$ \\
\hline 14 & $16: 51: 20.343$ & $35: 02: 32.628$ & 0.7936 & 135.97 & $8.68_{-0.30}^{+0.50}$ & $0.98_{-0.06}^{+0.08}$ & $0.21 \pm 0.04$ & 9.3 & $2.173 \pm 0.089$ & $7.209 \pm 0.040$ & $105.147_{-09.669}^{+12.086}$ & $4.07_{-0.02}^{+0.01}$ & $8.33 \pm 0.06$ \\
\hline 15 & 23:27:20.369 & 00:05:54.762 & 0.7553 & 741.71 & $8.13_{-0.21}^{+0.01}$ & $1.29_{-0.10}^{+0.09}$ & $0.48 \pm 0.05$ & 13.6 & $1.034_{-0.027}^{+0.096}$ & $7.428_{-0.042}^{+0.084}$ & $72.096_{-06.921}^{+04.614}$ & $4.15_{-0.02}^{+0.01}$ & $8.00_{-0.05}^{+0.04}$ \\
\hline 16 & $23: 27: 43.140$ & 00:12:42.832 & 0.7743 & 34.15 & $9.23_{-0.20}^{+0.22}$ & $0.71 \pm 0.42$ & $0.22 \pm 0.23$ & 3.4 & $1.958_{-0.000}^{+0.945}$ & $4.320 \pm 0.124$ & $46.844_{-12.492}^{+18.738}$ & $4.21 \pm 0.07$ & $7.85_{-0.18}^{+0.20}$ \\
\hline 17 & $23: 27: 29.854$ & 00:14:20.439 & 0.7637 & 40.62 & $8.39_{-0.01}^{+0.37}$ & $0.78 \pm 0.19$ & $0.32 \pm 0.10$ & 4.3 & $2.420_{-0.074}^{+0.596}$ & $6.508_{-0.074}^{+0.147}$ & $89.583_{-17.339}^{+28.898}$ & $4.09 \pm 0.04$ & $8.29_{-0.15}^{+0.13}$ \\
\hline 18 & 23:27:07.500 & $00: 17: 41.503$ & 0.7885 & 49.03 & $9.34_{-0.29}^{+0.21}$ & $0.96_{-0.17}^{+0.14}$ & $0.34_{-0.09}^{+0.08}$ & 4.4 & $1.794_{-0.194}^{+0.129}$ & $7.022 \pm 0.113$ & $115.500_{-14.903}^{+37.258}$ & $4.04 \pm 0.04$ & $8.38_{-0.15}^{+0.10}$ \\
\hline 19 & 23:26:55.430 & 00:17:52.919 & 0.8562 & 97.37 & $8.99_{-0.07}^{+0.00}$ & $0.62_{-0.08}^{+0.13}$ & $0.00_{-0.04}^{+0.07}$ & 5.2 & $1.636_{-0.026}^{+0.021}$ & $7.989_{-0.132}^{+0.066}$ & $128.952_{-23.991}^{+17.993}$ & $4.04_{-0.02}^{+0.03}$ & $8.40_{-0.13}^{+0.08}$ \\
\hline 20 & 23:30:57.949 & 00:03:38.191 & 0.7842 & 87.95 & $8.61_{-0.28}^{+0.07}$ & $0.66_{-0.26}^{+0.18}$ & $0.26_{-0.14}^{+0.10}$ & 4.1 & $2.017_{-0.221}^{+0.276}$ & $5.575_{-0.159}^{+0.132}$ & $36.499_{-05.840}^{+14.600}$ & $4.26_{-0.09}^{+0.05}$ & $7.72_{-0.19}^{+0.14}$ \\
\hline 21 & 23:31:50.728 & 00:09:39.393 & 0.8225 & 63.83 & $8.81_{-0.52}^{+0.06}$ & $0.34_{-0.30}^{+0.29}$ & $0.09_{-0.17}^{+0.16}$ & 5.0 & $1.692_{-0.115}^{+0.344}$ & $6.989_{-0.223}^{+0.179}$ & $52.462_{-08.507}^{+11.343}$ & $4.19_{-0.04}^{+0.05}$ & $7.93_{-0.14}^{+0.10}$ \\
\hline 22 & $02: 27: 48.871$ & 00:24:40.077 & 0.7838 & 298.74 & $7.85_{-0.06}^{+0.45}$ & $0.30_{-0.23}^{+0.16}$ & $0.07_{-0.13}^{+0.09}$ & 5.3 & $1.735_{-0.043}^{+0.343}$ & $7.110_{-0.207}^{+0.103}$ & $61.688_{-09.490}^{+12.654}$ & $4.16_{-0.04}^{+0.03}$ & $8.04_{-0.12}^{+0.10}$ \\
\hline 23 & 02:27:05.706 & $00: 25: 21.865$ & 0.7661 & 77.52 & $8.63_{-0.18}^{+0.07}$ & $0.63 \pm 0.42$ & $0.22 \pm 0.23$ & 3.2 & $1.492_{-0.000}^{+0.720}$ & $7.631_{-0.108}^{+0.135}$ & $144.017_{-57.607}^{+00.000}$ & $4.05_{-0.06}^{+0.05}$ & $8.41_{-0.20}^{+0.12}$ \\
\hline 24 & 02:27:30.457 & 00:31:06.391 & 0.7214 & 159.58 & $7.87_{-0.29}^{+0.52}$ & $0.90_{-0.06}^{+0.07}$ & $0.25 \pm 0.04$ & 9.6 & $1.525 \pm 0.076$ & $7.105 \pm 0.057$ & $94.916_{-10.430}^{+08.344}$ & $4.09 \pm 0.02$ & $8.20_{-0.06}^{+0.05}$ \\
\hline 25 & 02:26:03.707 & $00: 36: 22.460$ & 0.7888 & 66.11 & $8.69_{-0.48}^{+0.22}$ & $2.12_{-0.17}^{+0.20}$ & $1.02_{-0.09}^{+0.11}$ & 6.6 & $2.857_{-0.336}^{+0.269}$ & $4.878_{-0.065}^{+0.130}$ & $48.598_{-08.526}^{+05.116}$ & $4.23_{-0.03}^{+0.04}$ & $7.84_{-0.09}^{+0.10}$ \\
\hline 26 & 02:26:21.479 & 00:48:06.813 & 0.7743 & 36.94 & $9.24_{-0.29}^{+0.08}$ & $0.54 \pm 0.10$ & $0.00_{-0.06}^{+0.05}$ & 6.7 & $2.064_{-0.041}^{+0.031}$ & $5.895_{-0.044}^{+0.089}$ & $109.091 \pm 14.307$ & $4.07 \pm 0.02$ & $8.29_{-0.10}^{+0.08}$ \\
\hline 27 & 02:29:33.654 & 00:26:08.023 & 0.7294 & 67.55 & $8.66_{-0.67}^{+0.20}$ & $0.80_{-0.20}^{+0.14}$ & $0.36_{-0.11}^{+0.08}$ & 7.0 & $2.555_{-0.330}^{+0.220}$ & $7.849_{-0.231}^{+0.132}$ & $54.969_{-07.715}^{+09.644}$ & $4.19 \pm 0.04$ & $8.01_{-0.12}^{+0.09}$ \\
\hline 28 & 02:29:02.031 & 00:30:08.127 & 0.7315 & 99.97 & $8.70_{-0.06}^{+0.01}$ & $1.18_{-0.04}^{+0.03}$ & $0.30 \pm 0.02$ & 15.2 & $1.515_{-0.028}^{+0.042}$ & $7.633_{-0.019}^{+0.037}$ & $132.444_{-05.333}^{+10.667}$ & $4.04 \pm 0.01$ & $8.40_{-0.03}^{+0.04}$ \\
\hline
\end{tabular}

Note. Unless otherwise specified, $[\mathrm{O}$ III] refers to $[\mathrm{O}$ III] $\lambda \lambda 44959,5007$. 


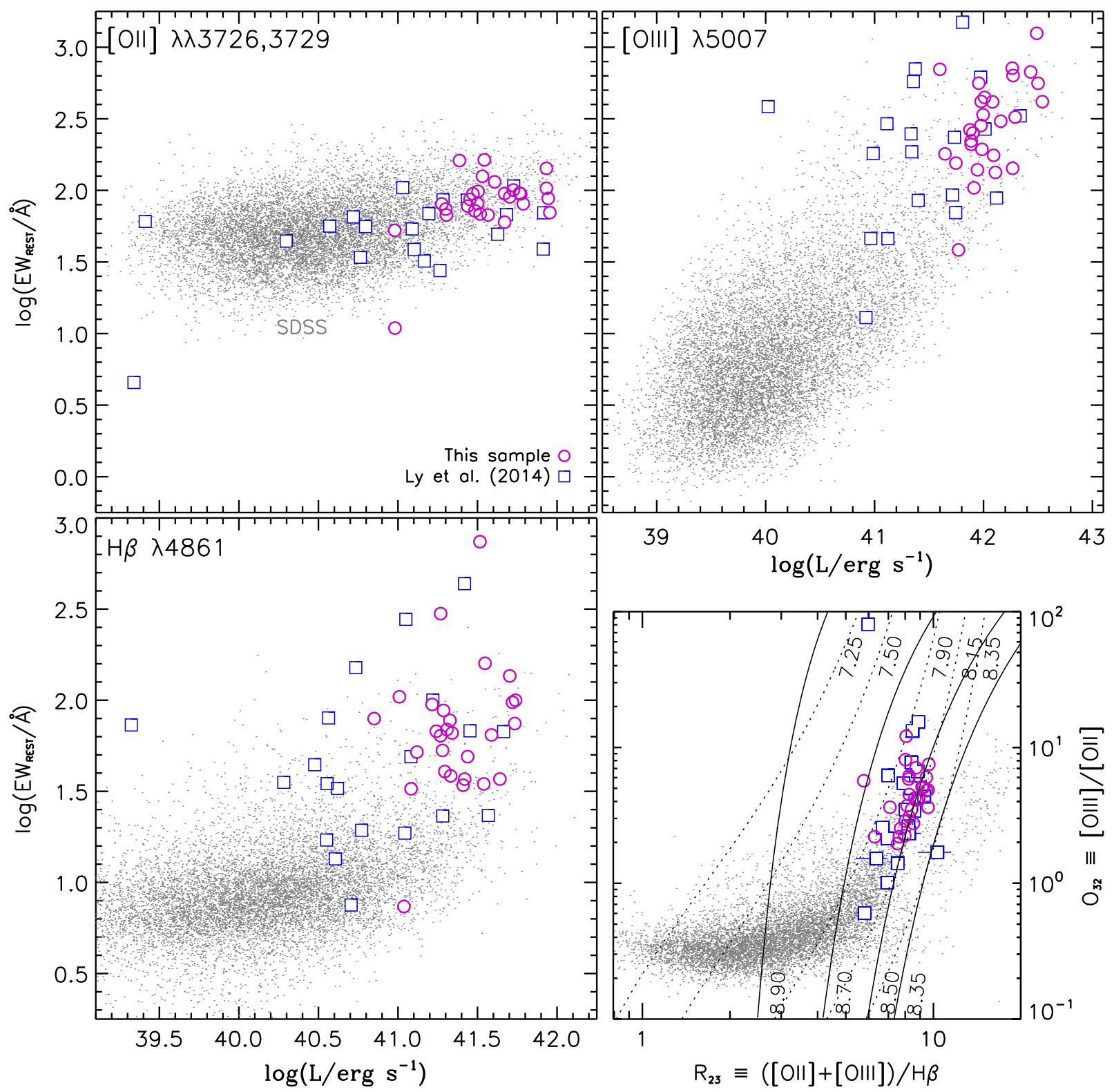

Figure 2. Emission-line luminosities, flux ratios, and rest-frame EWs for our $[\mathrm{O}$ III $] \lambda 4363$ sample (purple circles). All luminosities and flux ratios are observed, before correction for dust attenuation. Gray points illustrate the SDSS DR7 emission-line sample. The lower right panel shows the metallicity-sensitive $\left(R_{23}\right)$ and ionization parameter-sensitive $\left(O_{32}\right)$ emission-line ratios. Photoionization models from McGaugh $(1991)$ are overlaid for metallicities between $12+\log (\mathrm{O} / \mathrm{H})=7.25$ and $12+\log (\mathrm{O} / \mathrm{H})=8.9$. Solid (dotted) curves are for metallicities on the "upper" ("lower") $R_{23}$ branch. Overlaid as blue squares is the [O III] $\lambda 4363$-detected sample from Ly et al. (2014).

Assuming a Chabrier (2003) IMF with masses of 0.1-100 $M_{\odot}$ and solar metallicity, the SFR can be determined from the observed $\mathrm{H} \beta$ luminosity (Kennicutt 1998):

$$
\frac{\mathrm{SFR}}{M_{\odot} \mathrm{yr}^{-1}}=4.4 \times 10^{-42} \times 2.86 \times 10^{0.4 A(\mathrm{H} \beta)} \frac{L(\mathrm{H} \beta)}{\mathrm{erg} \mathrm{s}^{-1}},
$$

where $A(\mathrm{H} \beta)=4.6 E(B-V)$. This relation overestimates the SFR at low metallicities due to the dependence of a stronger ionizing radiation field on lower metallicity. Since our galaxies have $Z \approx 0.2 Z_{\odot}$, we reduce the SFRs by $37 \%$ (Henry et al. 2013b). Our SFR estimates are summarized in Table 1 and are illustrated in Figure 5. We find that our galaxies have dust-corrected SFRs of $0.8-130 M_{\odot} \mathrm{yr}^{-1}$ with an average (median) of $10.7(4.6) M_{\odot} \mathrm{yr}^{-1}$.

\subsection{Stellar Masses from SED Modeling}

To determine stellar masses, we follow the common approach of modeling the spectral energy distribution (SED) with stellar synthesis models (e.g., Salim et al. 2007; Ly et al. 2011, 2012b). The eight-band photometric data include BRI imaging from the Canada-France-Hawaii Telescope (CFHT) for the DEEP2 survey (Coil et al. 2004). In addition, publicly available ugriz imaging from the CFHT Legacy Survey is available in Field \#1 (Extended Groth Strip), and Fields \#3-4 are located in the SDSS deep survey strip (Stripe 82) for $u^{\prime} g^{\prime} r^{\prime} i^{\prime} z^{\prime}$ imaging. Thus, 18 of 27 galaxies have eight optical imaging bands. These photometric data that we use have been compiled by Matthews et al. (2013). While our galaxies have low stellar masses (as demonstrated below), the imaging data are fairly deep. The $R$-band imaging, for example, 


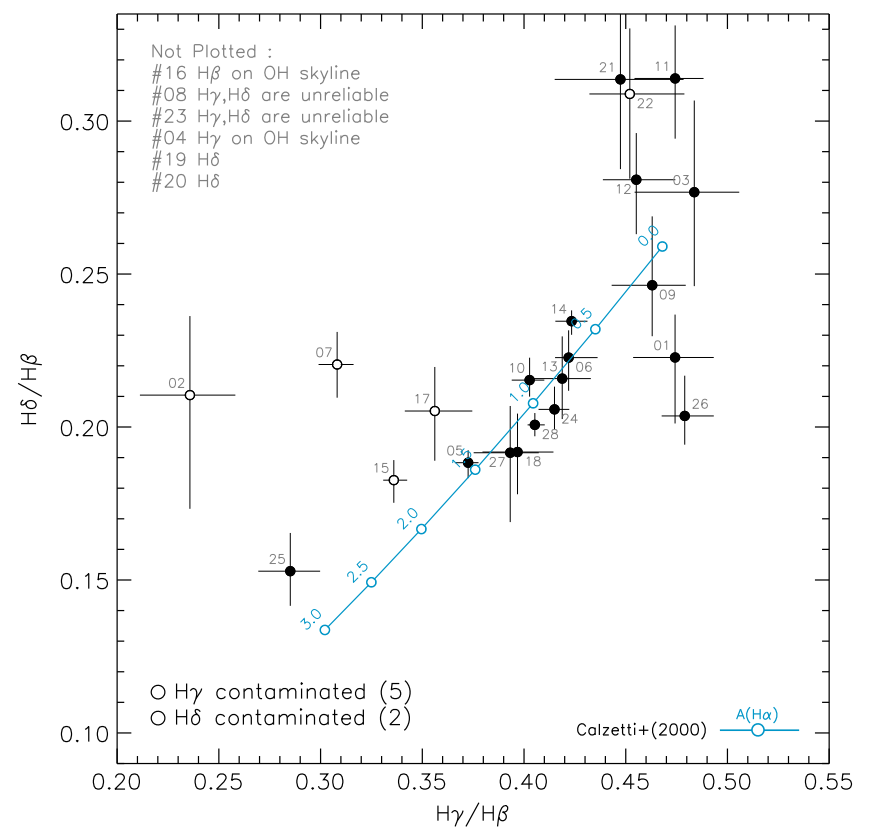

Figure 3. Balmer decrements $(\mathrm{H} \gamma / \mathrm{H} \beta$ and $\mathrm{H} \delta / \mathrm{H} \beta)$ for our $[\mathrm{O}$ III] $\lambda 4363$ sample. Reliable measurements are shown by the filled circles, while those affected by contamination from $\mathrm{OH}$ sky-line emission are shown as open circles. Blue circles and curve show the effects on the Balmer decrements with increasing dust reddening following Calzetti et al. (2000). Values adjacent to blue circles indicate $A(\mathrm{H} \alpha)$. The significant scatter in the upper right is due to less reliable $\mathrm{H} \delta / \mathrm{H} \beta$ measurements. These galaxies all have $\mathrm{H} \gamma / \mathrm{H} \beta$ measurements that are consistent with $A(\mathrm{H} \alpha) \sim 0$.

reaches an $8 \sigma \mathrm{AB}$ limit of 24.5 (Newman et al. 2013), and our galaxies are on average $1.2 \mathrm{AB}$ mag brighter than this limit.

To extend the wavelength coverage, we cross-matched our sample against the catalog of Bundy et al. (2006), which contains $J K$ photometry. Unfortunately, only two galaxies have a match. This is not a surprise since many of our galaxies have low stellar masses. While photometric data redward of $5500 \AA$ are unavailable, Zahid et al. (2011) demonstrated that stellar mass estimates from $B R I$ photometry are consistent with those obtained from $B R I K_{s}$, suggesting that the existing optical data are sufficient for stellar mass estimates. We also note that the lack of ugriz imaging does not significantly hamper out SED modeling for nine galaxies, as we compared stellar mass estimates derived from $B R I$-only and $B R I+$ ugriz photometry for two-thirds of our sample and find consistent results. Future efforts will include acquiring Spitzer infrared data to provide more robust stellar mass estimates.

To model the SED, we use the Fitting and Assessment of Synthetic Templates (FAST) code (Kriek et al. 2009) with Bruzual \& Charlot (2003) models and adopt a Chabrier (2003) IMF, exponentially declining star formation histories (i.e., $\tau$ models), one-fifth solar metallicity, and Calzetti et al. (2000) reddening. We also correct the broadband photometry for the contribution of nebular emission lines following the approach described in Ly et al. (2014). This correction reduces the stellar mass estimates by 0.2 dex (average). To determine stellar mass uncertainties, we conduct Monte Carlo realizations within FAST. Here, data points are randomly perturbed 100 times (based on the photometric uncertainties) and the SEDs are refitted, yielding a probability distribution function for stellar mass. The stellar masses are provided in Table 1 and are illustrated in Figures 4-5. The average (median) stellar masses are $4.9 \times 10^{8} \quad M_{\odot}\left(5.0 \times 10^{8} M_{\odot}\right)$ and span $7.1 \times 10^{7}-$
$2.2 \times 10^{9} M_{\odot}$.

\section{RESULTS}

\subsection{Excitation Properties}

Figure 4 illustrates the $[\mathrm{O}$ III $] \lambda 5007 / \mathrm{H} \beta$ flux ratios and stellar masses along the "Mass-Excitation" (MEx; Juneau et al. 2014) diagram. The MEx is used as a substitute for the Baldwin et al. (1981) diagnostic diagram when [N II] $\lambda 6583 / \mathrm{H} \alpha$ is unavailable. It can be seen that these galaxies have high $[\mathrm{O} \mathrm{III}] / \mathrm{H} \beta$ ratios, $5.0 \pm 0.9$. All of them are classified as star-forming galaxies by falling below the solid black line. Compared to other metal-poor galaxies (Ly et al. 2014; blue squares), the DEEP2 galaxies have similar excitation properties, but are $\approx 0.4$ dex more massive. Compared to UV- and mass-selected $z \sim 2$ galaxies (e.g., Shapley et al. 2015; Steidel et al. 2014), our measured $[\mathrm{O} \quad \mathrm{III}] / \mathrm{H} \beta$ ratios are higher by a factor of 1.25-2.5. Their strong-line oxygen ratios, $R_{23}$ and $O_{32}$, are consistent with $z \sim 2$ galaxies from Shapley et al. (2015).

\subsection{Relationship between Mass, Metallicity, and SFR}

Figure 5(a) compares the dust-corrected instantaneous SFRs against the stellar mass estimates. Here we compare our work against mass-selected galaxies at $z \sim 1$ (Whitaker et al. 2014b) and $\mathrm{H} \alpha$-selected galaxies at $z \approx 0.8$ (de los Reyes et al. 2015). Our galaxies are located $1.05 \pm 0.61$ dex above these $M_{\star}-\mathrm{SFR}$ relations with $\mathrm{SFR} / M_{\star}$ of $10^{-8.0 \pm 0.6} \mathrm{yr}^{-1}$. This significant SFR offset is also seen for metal-poor galaxies from Ly et al. (2014). By requiring [O III] $\lambda 4363$ detections, both $[\mathrm{O}$ III] $\lambda 4363$ studies are biased toward high-EW emission lines (see Figure 2), which correspond to higher sSFRs.

Figure 5(c) illustrates the $M_{\star}-Z$ relation. Here we compare our results against Andrews \& Martini (2013). It demonstrates that while a subset of our galaxies is consistent with Andrews \& Martini (2013), a significant fraction (60\%) are located below the relation at more than $0.22 \mathrm{dex}(1 \sigma$; Andrews \& Martini 2013), by as much as -0.76 dex. This results in an average $Z$ offset for the sample of $-0.28 \pm 0.23$ dex. Our $M_{\star}-Z$ relation result is consistent with Ly et al. (2014) (blue squares), who also found that half of their sample falls below the local $M_{\star}-Z$ relation.

The FMR was introduced to describe the dependence between $M_{\star}, Z$, and SFR in local galaxies and was extended to explain higher-redshift galaxies. Mannucci et al. (2010) was one of the first studies to parameterize this dependence by considering a combination of stellar mass and SFR:

$$
\mu=\log \left(M_{\star}\right)-\alpha \log (\mathrm{SFR}),
$$

where $\alpha$ is the coefficient that minimizes the scatter against metallicity. Figure 5(e) illustrates the $\mu$ projection of the $M_{\star}-$ $Z-S F R$ relation with $\alpha=0.66$ (Andrews \& Martini 2013). It can be seen that our sample is consistent $(0.01 \pm 0.29 \mathrm{dex})$ with the local FMR; however, significant dispersion remains. The dispersion is greater than our $M_{\star}-Z$ comparison and the average measurement uncertainties of $\approx 0.16 \mathrm{dex}$ with respect to the FMR.

The local $M_{\star}-Z-$ SFR relation suggests that higher SFR galaxies have lower metallicity at fixed stellar mass. To examine if this is correct, we split our sample by high and low sSFRs and perform Kolmogorov-Smirnov (K-S) tests to 


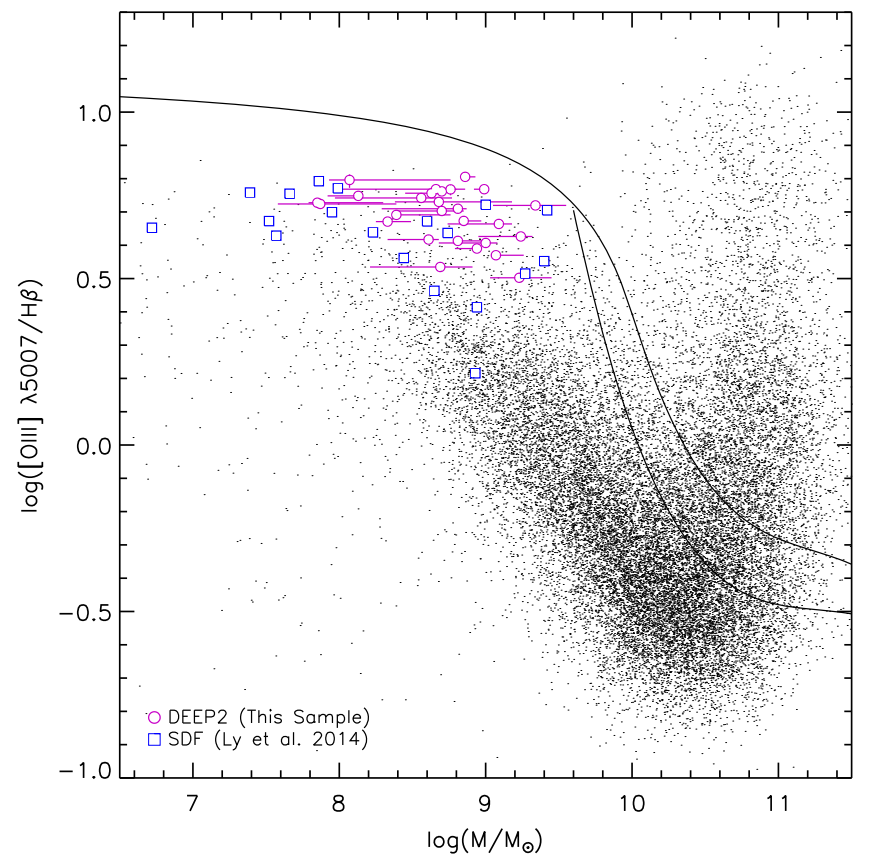

Figure 4. $[\mathrm{O}$ III $] / \mathrm{H} \beta$ flux ratio ("excitation") as a function of stellar mass (i.e., the MEx diagram; Juneau et al. 2014). The DEEP2 [O III] $\lambda 4363$ sample is shown by the purple circles. Overlaid as blue squares is the Ly et al. (2014) [O III] $\lambda 4363$ sample from the SDF. The SDSS DR7 sample is illustrated in black.

determine if these two distributions are different. These two samples are illustrated in panels (b), (d), and (f) in Figure 5 as filled (high-SFR) and open (low-SFR) symbols. The sample is divided at the median $\Delta[\log (\mathrm{SFR})]$, which is the amount of deviation relative to the Whitaker et al. (2014a) $M_{\star}-\mathrm{SFR}$ relation. This relative SFR offset follows the non-parametric approach of Salim et al. (2014).

One concern with conducting a K-S test is the selection bias of requiring the detection of [O III] $\lambda 4363$. More specifically, detection of this line primarily depends on the electron temperature (or gas metallicity), which corresponds to the $\lambda 5007 / \lambda 4363$ line ratio, and the dust-corrected SFR, which determines the overall normalization of the emission-line strengths. At high SFRs, the probability of detecting [O $\mathrm{OII}]$ $\lambda 4363$ is greater for a wide range of metallicity. This range of metallicity reduces toward lower metallicity such that only metal-poor galaxies with low SFRs can be detected in an emission-line flux-limited survey.

This selection bias is demonstrated in Figure 6, which illustrates the gas-phase metallicity as a function of dustcorrected SFR. The black curves correspond to the average $\left[\begin{array}{ll}\mathrm{O} & \mathrm{III}\end{array}\right] \lambda 4363 \mathrm{~S} / \mathrm{N}=3$ detection limit of $3.4 \times 10^{-18}$ $\mathrm{erg} \mathrm{s}^{-1} \mathrm{~cm}^{-2}$. This limit was determined from measuring the $\mathrm{rms}$ in 4140 spectra in areas adjacent to where [O $\mathrm{III}] \lambda 4363$ is expected to be detected. In determining the metallicity-SFR dependence, we consider (1) an ionic oxygen abundance ratio $\left(\mathrm{O}^{++} / \mathrm{O}^{+}\right)$of unity, which is the average for our DEEP2 [O III] $\lambda 4363$ sample, and (2) a temperature-metallicity relation of $12+\log (\mathrm{O} / \mathrm{H})=9.51-1.03\left(T_{e} / 10^{4} \mathrm{~K}\right)$. The latter is empirically determined from our DEEP $2[\mathrm{O}$ III $\lambda 4363$ galaxies.

To account for the $Z$-SFR selection bias, we include reliable [O III $] \lambda 4363$ non-detections within our K-S analyses. First, we consider all DEEP2 galaxies with $\mathrm{S} / \mathrm{N} \geqslant 100$ on $[\mathrm{O}$ III] $\lambda 5007$ and a non-detection $(\mathrm{S} / \mathrm{N}<3)$ on $[\mathrm{O}$ III $] \lambda 4363$. This sample of
126 galaxies is then vetted for unreliable limits because the [O III] $\lambda 4363$ emission line either falls on an $\mathrm{OH}$ skyline, the atmospheric A band, or a CCD gap. This limited the sample to 79 galaxies. While the above [O III] $\lambda 5007 \mathrm{~S} / \mathrm{N}$ cut is strict, for many of these galaxies, a strong lower limit on the metallicity is not available. This is because an $\mathrm{S} / \mathrm{N}=100$ corresponds roughly to 0.03 on $\lambda 4363 / \lambda 5007$ or $T_{e} \approx 2 \times 10^{4} \mathrm{~K}$. Thus, we further restrict our non-detection sample to 13 galaxies with $[\mathrm{O}$ III $] \lambda 5007 \mathrm{~S} / \mathrm{N} \geqslant 200$. These galaxies are overlaid in Figure 6 and panels (b), (d), and (f) of Figure 5 as either black circles or black triangles $(\mathrm{S} / \mathrm{N}=3$ lower limit on metallicity). It can be seen that the majority of these galaxies are located to the left of the $\mathrm{S} / \mathrm{N}=3$ line for $E(B-V)=0.22 \mathrm{mag}$ in Figure 6. Also, Figure 5 illustrates that these galaxies have higher stellar masses $(0.3$ dex $)$ than our detected sample and lower SFRs at a given stellar mass. As expected, these galaxies have higher metallicity with lower limits above $12+\log (\mathrm{O} / \mathrm{H}) \approx 8.2$ (average: 8.37).

For our $\mathrm{K}-\mathrm{S}$ tests, we compare the $\log (\mathrm{O} / \mathrm{H})$ distributions for the low- and high-SFR samples, finding that these two distributions are similar with the lower SFR galaxies having slightly higher metallicity (see Figure $7(\mathrm{a})$ ). However, as Figure 5(b) shows, these two samples differ in stellar mass by $\approx 0.5$ dex. If instead we consider the relative offset in metallicity against the $M_{\star}-Z$ relation of Andrews \& Martini (2013), the K-S test finds that the two samples are different at $94.4 \%(1.9 \sigma$; Figure $7(\mathrm{~b}))$. The difference, however, is in the opposite direction of local predictions, with higher sSFR galaxies having higher gas-phase metallicities.

Given this discrepancy, it is also important to investigate whether the same result is seen when considering the FMR that Mannucci et al. (2010) defined. Here, they utilized the $R_{23}$ strong-line diagnostic and a fourth-order polynomial for $R_{23}-Z$ (Maiolino et al. 2008). However, the majority of our DEEP2 sample has dust-corrected $R_{23}$ values that exceed the maximum threshold value of $R_{23}=8.65$. Thus, it is not possible to conduct the same $\mathrm{K}-\mathrm{S}$ test analysis with $R_{23}$ and the Maiolino et al. (2008) calibration.

\section{DISCUSSIONS}

From DEEP2 spectra of 28 galaxies with oxygen abundances from $[\mathrm{O} \mathrm{III}] \lambda 4363$ detections (i.e., the $T_{e}$ method), we find that metal-poor strongly star-forming galaxies are consistent with the local FMR (Andrews \& Martini 2013), albeit with large dispersion $(0.29$ dex with 0.16 dex due to measurement errors). This result is consistent with metal-poor galaxies from Ly et al. (2014) and lensed low-mass starforming galaxies at $z \sim 0.8-2.6$ (Wuyts et al. 2012). Given the high sSFRs of $\sim(100 \mathrm{Myr})^{-1}$, we argue that the large dispersion in metallicity is unsurprising-these galaxies are most likely undergoing episodic star formation and have not settled into a steady state.

We find marginal $(94.4 \% ; 1.9 \sigma)$ evidence that galaxies with higher sSFRs $\left(\lesssim 10^{-8} \mathrm{yr}^{-1}\right)$ are more metal-rich. While this contradicts previous local studies, the inverse of the sSFR - timescale for star formation-is short. Assuming outflow velocities comparable to virial velocities $(\sim 150 \mathrm{~km}$ $\left.\mathrm{s}^{-1}\right)$ for $\log \left(M_{\text {halo }} / M_{\odot}\right) \approx 11.1$ (Behroozi et al. 2010), eight galaxies in our sample would not have enough time $\left(\mathrm{sSFR}^{-1} \lesssim 10^{7.65} \mathrm{yr}\right)$ for any recently enriched outflows to be driven out of the $1^{\prime \prime}(7.5 \mathrm{kpc})$ slit widths. Thus, one would expect the SFR- $Z$ dependence to turn positive for low-mass 

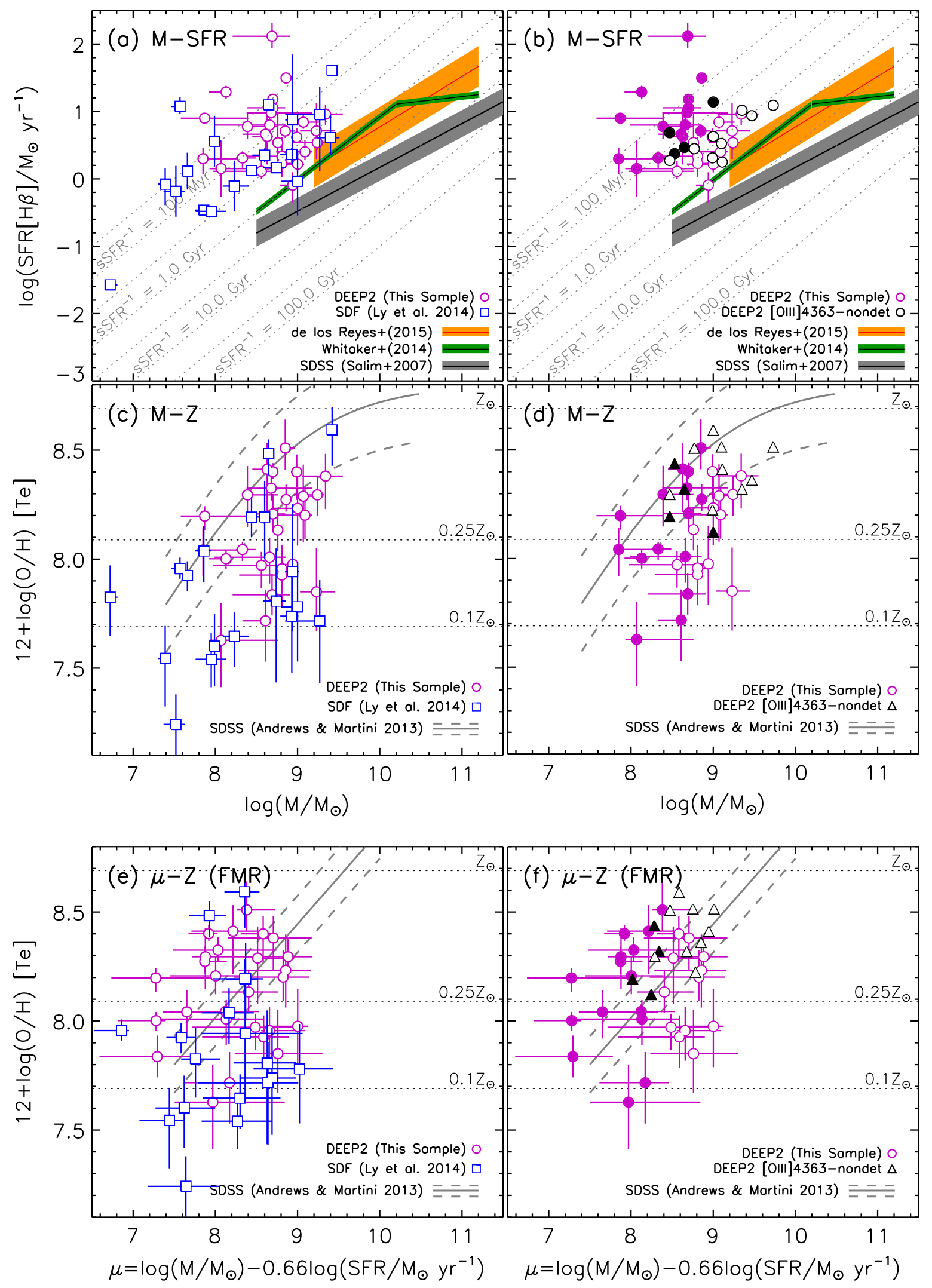

Figure 5. Relations between stellar mass and (top) dust-corrected $\mathrm{H} \beta$ SFR, (middle) metallicity, and (bottom) metallicity and dust-corrected $\mathrm{H} \beta$ SFR (i.e., the FMR). The DEEP2 [O III] $\lambda 4363$ sample is shown by the purple circles. Overlaid as blue squares on the left panels is the Ly et al. (2014) [O III] $\lambda 4363$ sample from the SDF. Results from the SDSS sample (Salim et al. 2007; Andrews \& Martini 2013) are illustrated in gray. For comparisons, we also overlay in the top panels the stellar mass-SFR relation of $\mathrm{H} \alpha$-selected galaxies at $z=0.8$ (de los Reyes et al. 2015) and mass-selected star-forming galaxies at $z=0.5-1$ (Whitaker et al. 2014a) in orange and green, respectively. For direct metallicity comparisons, we illustrate the results of Andrews \& Martini (2013), which stacked spectra to measure average $T_{e}$-based metallicities. DEEP2 galaxies with reliable non-detections of [O III] $\lambda 4363$ are shown as black circles and triangles (lower limit on metallicity). The DEEP2 samples are separated by low and high SFRs (see text) as open and filled symbols, respectively. 


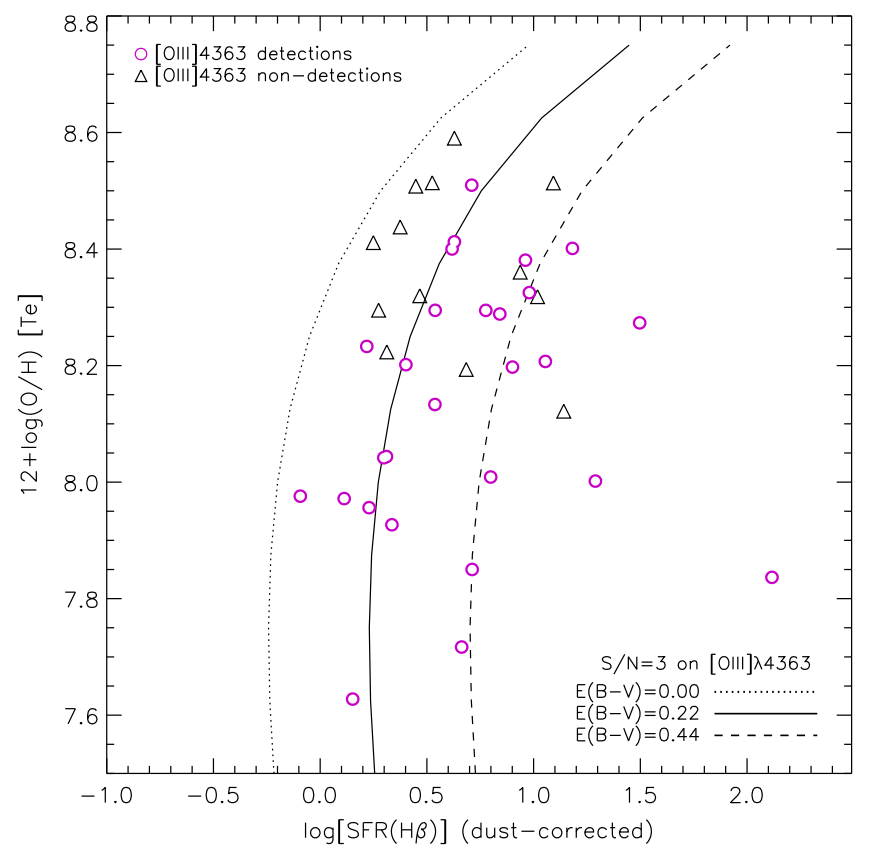

Figure 6. Gas-phase metallicity as a function of dust-corrected SFR for our $[\mathrm{O}$ III] $\lambda 4363$ sample (purple circles) and a sample of reliable [O $\mathrm{OII}] \lambda 4363$ non-detections at $\mathrm{S} / \mathrm{N}<3$ (black triangles). The triangles are lower limits on metallicity. The dotted, solid, and dashed lines correspond to an $\mathrm{S} / \mathrm{N}=3$ limit on $\left[\mathrm{O}\right.$ III] $\lambda 4363$ of $3.4 \times 10^{-18} \mathrm{erg} \mathrm{s}^{-1} \mathrm{~cm}^{-2}$ for three dust extinction possibilities, $E(B-V)=0.0,0.22$, and 0.44 , respectively. These extinction values span the dispersion seen in our sample.

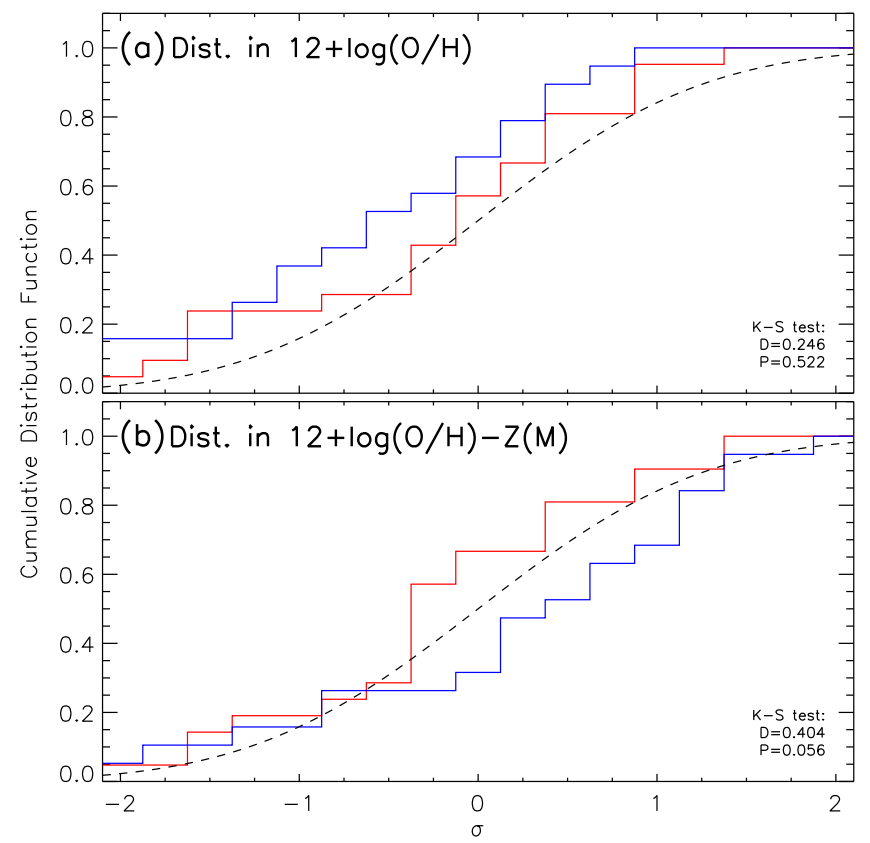

Figure 7. Cumulative distribution functions in (a) metallicity and (b) metallicity relative to the Andrews \& Martini (2013) $M_{\star}-Z$ relation, for two samples with low (red line) and high SFRs (blue line). The $\mathrm{K}-\mathrm{S}$ statistics $(D)$ and the probability that the two distributions are identical $(P)$ are given in the lower right-hand corner. Panel (b) shows that the two distributions are different at $94.4 \%$ confidence.

strongly star-forming galaxies. Given the instantaneous SFRs, we find that the measured oxygen abundances can be explained with low nucleosynthesis yields $(y \sim 0.01)$, gas-to- stellar mass fraction of $\approx 1 \pm 0.4$, and no metal loss due to outflows.

Based on observations taken at the W. M. Keck Observatory, which is operated jointly by the National Aeronautics and Space Administration (NASA), the University of California, and the California Institute of Technology. Funding for the DEEP2 Galaxy Redshift Survey has been provided by NSF grants AST-9509298, AST-0071048, AST-0507428, and AST0507483, as well as NASA LTSA grant NNG04GC89G. C.L. is funded through the NASA Postdoctoral Program. We thank Jeffrey Newman, Alaina Henry, Massimo Ricotti, and Kate Whitaker for insightful discussions, and the anonymous referee for insightful comments that improved the paper.

\section{REFERENCES}

Aller, L. H. 1984, Astrophysics and Space Science Library (Reidel: Dordrecht) Amorín, R., Pérez-Montero, E., Contini, T., et al. 2014a, A\&A, submitted (arXiv:1403.3441)

Amorín, R., Sommariva, V., Castellano, M., et al. 2014b, A\&A, 568, LL8

Andrews, B. H., \& Martini, P. 2013, ApJ, 765, 140

Atek, H., Siana, B., Scarlata, C., et al. 2011, ApJ, 743, 121

Baldwin, A., Phillips, M. M., \& Terlevich, R. 1981, PASP, 93, 817

Berg, D. A., Skillman, E. D., Marble, A. R., et al. 2012, ApJ, 754, 98

Behroozi, P. S., Conroy, C., \& Wechsler, R. H. 2010, ApJ, 717, 379

Brown, W. R., Kewley, L. J., \& Geller, M. J. 2008, AJ, 135, 92

Bruzual, G., \& Charlot, S. 2003, MNRAS, 344, 1000

Bundy, K., Ellis, R. S., Conselice, C. J., et al. 2006, ApJ, 651, 120

Calzetti, D., Armus, L., Bohlin, R. C., et al. 2000, ApJ, 533, 682

Chabrier, G. 2003, PASP, 115, 763

Coil, A. L., Newman, J. A., Kaiser, N., et al. 2004, ApJ, 617, 765

Davis, M., Faber, S. M., Newman, J., et al. 2003, Proc. SPIE, 4834, 161

de los Reyes, M., Ly, C., \& Lee, J. C. 2015, AJ, 149, 79

Domínguez, A., Siana, B., Henry, A. L., et al. 2013, ApJ, 763, 145

Ellison, S. L., Patton, D. R., Simard, L., \& McConnachie, A. W. 2008, ApJL, 672, L107

Erb, D. K., Shapley, A. E., Pettini, M., et al. 2006, ApJ, 644, 813

Faber, S. M., Phillips, A. C., Kibrick, R. I., et al. 2003, Proc. SPIE, 4841, 1657

Hainline, K. N., Shapley, A. E., Kornei, K. A., et al. 2009, ApJ, 701, 52

Hayashi, M., Motohara, K., Shimasaku, K., et al. 2009, ApJ, 691, 140

Henry, A., Martin, C. L., Finlator, K., \& Dressler, A. 2013a, ApJ, 769, 148

Henry, A., Scarlata, C., Domínguez, A., et al. 2013b, ApJ, 776, L27

Hunt, L., Magrini, L., Galli, D., et al. 2012, MNRAS, 427, 906

Hoyos, C., Koo, D. C., Phillips, A. C., Willmer, C. N. A., \& Guhathakurta, P. 2005, ApJL, 635, L21

Hu, E. M., Cowie, L. L., Kakazu, Y., \& Barger, A. J. 2009, ApJ, 698, 2014 Izotov, Y. I., Stasińska, G., Meynet, G., Guseva, N. G., \& Thuan, T. X. 2006, A\&A, 448, 955

Izotov, Y. I., Thuan, T. X., \& Guseva, N. G. 2012, A\&A, 546, A122

Juneau, S., Bournaud, F., Charlot, S., et al. 2014, ApJ, 788, 88

Kakazu, Y., Cowie, L. L., \& Hu, E. M. 2007, ApJ, 668, 853

Kashikawa, N., Shimasaku, K., Yasuda, N., et al. 2004, PASJ, 56, 1011

Kennicutt, R. C. 1998, ARA\&A, 36, 189

Kewley, L. J., Dopita, M. A., Leitherer, C., et al. 2013a, ApJ, 774, 100

Kewley, L. J., Maier, C., Yabe, K., et al. 2013b, ApJL, 774, L10

Kobulnicky, H. A., \& Kewley, L. J. 2004, ApJ, 617, 240

Kriek, M., van Dokkum, P. G., Labbé, I., et al. 2009, ApJ, 700, 221

Lara-López, M. A., Cepa, J., Bongiovanni, A., et al. 2010, A\&A, 521, L53

Liu, X., Shapley, A. E., Coil, A. L., Brinchmann, J., \& Ma, C.-P. 2008, ApJ, 678,758

Ly, C., Malkan, M. A., Hayashi, M., et al. 2011, ApJ, 735, 91

Ly, C., Malkan, M. A., Kashikawa, N., et al. 2007, ApJ, 657, 738

Ly, C., Malkan, M. A., Kashikawa, N., et al. 2012a, ApJL, 747, L16

Ly, C., Malkan, M. A., Kashikawa, N., et al. 2012b, ApJ, 757, 63

Ly, C., Malkan, M. A., Nagao, T., et al. 2014, ApJ, 780, 122

Maiolino, R., Nagao, T., Grazian, A., et al. 2008, A\&A, 488, 463

Mannucci, F., Cresci, G., Maiolino, R., et al. 2009, MNRAS, 398, 1915

Mannucci, F., Cresci, G., Maiolino, R., Marconi, A., \& Gnerucci, A. 2010 , MNRAS, 408, 2115

Markwardt, C. B. 2009, in ASP Conf. Ser. 411, Astronomical Data Analysis Software and Systems XVIII, ed. D. A. Bohlender, D. Durand, \& P. Dowler (San Francisco, CA: ASP), 251 
Matthews, D. J., Newman, J. A., Coil, A. L., Cooper, M. C., \& Gwyn, S. D. J. 2013, ApJS, 204, 21

McGaugh, S. S. 1991, ApJ, 380, 140

Momcheva, I. G., Lee, J. C., Ly, C., et al. 2013, AJ, 145, 47

Moustakas, J., Zaritsky, D., Brown, M., et al. 2011, ApJ, submitted (arXiv:1112.3300)

Nakajima, K., Ouchi, M., Shimasaku, K., et al. 2012, ApJ, 745, 12

Newman, J. A., Cooper, M. C., Davis, M., et al. 2013, ApJS, 208, 5

Nicholls, D. C., Dopita, M. A., Sutherland, R. S., Jerjen, H., \& Kewley, L. J. 2014, ApJ, 790, 75

Nicholls, D. C., Dopita, M. A., Sutherland, R. S., Kewley, L. J., \& Palay, E. 2013, ApJS, 207, 21

Pagel, B. E. J., Edmunds, M. G., Blackwell, D. E., Chun, M. S., \& Smith, G. 1979, MNRAS, 189, 95

Pirzkal, N., Rothberg, B., Ly, C., et al. 2013, ApJ, 772, 48
Rigby, J. R., Wuyts, E., Gladders, M. D., Sharon, K., \& Becker, G. D. 2011, ApJ, 732, 59

Salim, S., Lee, J. C., Ly, C., et al. 2014, ApJ, 797, 126

Salim, S., Rich, R. M., Charlot, S., et al. 2007, ApJS, 173, 267

Seaton, M. J. 1954, MNRAS, 114, 154

Shapley, A. E., Reddy, N. A., Kriek, M., et al. 2015, ApJ, 801, 88

Steidel, C. C., Rudie, G. C., Strom, A. L., et al. 2014, ApJ, 795, 165

Tremonti, C. A., Heckman, T. M., Kauffmann, G., et al. 2004, ApJ, 613, 898

Troncoso, P., Maiolino, R., Sommariva, V., et al. 2014, A\&A, 563, AA58

van der Wel, A., Straughn, A. N., Rix, H.-W., et al. 2011, ApJ, 742, 111

Whitaker, K. E., Franx, M., Leja, J., et al. 2014a, ApJ, 795, 104

Whitaker, K. E., Rigby, J. R., Brammer, G. B., et al. 2014b, ApJ, 790, 143

Wuyts, E., Rigby, J. R., Sharon, K., \& Gladders, M. D. 2012, ApJ, 755, 73

Xia, L., Malhotra, S., Rhoads, J., et al. 2012, AJ, 144, 28

Zahid, H. J., Kewley, L. J., \& Bresolin, F. 2011, ApJ, 730, 137 